\title{
Retrospective study of regorafenib and trifluridine/tipiracil efficacy as a third-line or later chemotherapy regimen for refractory metastatic colorectal cancer
}

\author{
AKIRA TANAKA, SOTARO SADAHIRO, TOSHIYUKI SUZUKI, \\ KAZUTAKE OKADA, GOTA SAITO and HIROSHI MIYAKITA \\ Department of Surgery, Tokai University School of Medicine, Isehara, Kanagawa 259-1193, Japan
}

Received March 21, 2018; Accepted August 30, 2018

DOI: $10.3892 / 01.2018 .9421$

\begin{abstract}
Regorafenib and trifluridine/tipiracil (TAS-102) are novel antitumor agents for patients with refractory metastatic colorectal cancer. However, it is unclear which patients may derive a survival benefit from these drugs in real-life clinical practice. We evaluated retrospectively the efficacy and safety of regorafenib and TAS-102 at a single institution between June 2013 and November 2015. Cox regression analysis was carried out to obtain predictive scores (the nearest integers of hazard ratio) for survival benefit. Forty-four patients treated with regorafenib or TAS-102 were included in the analysis; among them, 17 received crossover treatment. The median overall survival (OS) was 9.1 months for regorafenib and 9.3 months for TAS-102, and the corresponding values after crossover were 7.1 and 5.3 months, respectively. OS was not correlated to relative dose intensity, but was proportional to the total administered dose of each drug. Adverse events were tolerable even after crossover. We identified three variables as significant for prediction of OS with good discrimination $(C$-statistic $=0.70)$ : Poor Eastern Cooperative Oncology Group performance status, time since diagnosis of metastatic disease $\leq 18$ months, and previous chemotherapy continued $\geq 2$ months beyond progression were all predictors of poor OS. Regorafenib and TAS-102 can be recommended for patients with better performance status and slow progression of metastatic disease.
\end{abstract}

Correspondence to: Dr Akira Tanaka, Department of Surgery, Tokai University School of Medicine, 143 Shimokasuya, Isehara, Kanagawa 259-1193, Japan

E-mail: atanaka@tsc.u-tokai.ac.jp

Abbreviations: TAS-102, trifluridine and tipiracil; mCRC, metastatic colorectal cancer; VEGF, vascular endothelial growth factor; EGFR, epidermal growth factor receptor; KRAS, Kirsten rat sarcoma; CR, complete response; PR, partial response; SD, stable disease; PD, progressive disease; RECIST, Response Evaluation Criteria in Solid Tumors; CTCAE, Common Terminology Criteria for Adverse Events

Key words: salvage-line chemotherapy, prognostic score, regorafenib, trifluridine/tipiracil, colorectal cancer
Optimal survival benefit was provided by prompt administration of either drug after failure of previous chemotherapy, with flexible titration to the optimal dose for each individual patient.

\section{Introduction}

Colorectal cancer is the fourth most common cancer diagnosed in the United States, accounting for $8 \%$ of all new cancer cases (1). It is estimated that there were 132,700 new cases of colorectal cancer, and an estimated 49,700 people died of this disease in the US in 2015. Among 4,877 patients who received first-line chemotherapy for metastatic colorectal cancer (mCRC), identified in a nationwide and commercially available chemotherapy order entry system from 2004 to 2011, $53 \%(n=2,575)$ received second-line treatment, $28 \%(n=1,373)$ received third-line treatment, and only $13 \%(\mathrm{n}=640)$ received fourth-line treatment (2). Patients with mCRC infrequently go on to receive third-line or later treatment, and this might negatively impact on their overall survival (OS).

Regorafenib (Stivarga ${ }^{\circledR}$, Bayer AG, Leverkusen, Germany) is an oral multikinase inhibitor that blocks the activity of several protein kinases associated with angiogenesis [vascular endothelial growth factor (VEGF) receptors 1-3 and TIE2], oncogenesis (KIT, RET, RAF1 and BRAF), and the tumor microenvironment (PDGF receptor and FGF receptor) (3).

Trifluridine/tipiracil (TAS-102; Lonsurf ${ }^{\circledR}$; Taiho Pharmaceutical Co. Ltd, Tokyo) is an orally administered combination of a thymidine-based nucleic acid analogue, trifluridine, and a thymidine phosphorylase inhibitor, tipiracil hydrochloride. Trifluridine is the active cytotoxic component of TAS-102; its triphosphate form is incorporated into DNA, and this appears to result in its antitumor effects (4). Tipiracil hydrochloride is a potent inhibitor of thymidine phosphorylase, and serves to prevent the rapid degradation of trifluridine in TAS-102, providing more prolonged maintenance of adequate plasma levels of the active drug.

Regorafenib and TAS-102 are new salvage-line treatment options $(5,6)$, which provided statistically significant improvements of OS, progression-free survival (PFS), and disease control in placebo-controlled randomized phase III trials (CORRECT (7), CONCUR (8), RECOURSE (9) and TERRA) (10). Despite this evidence, these two drugs are often 
considered not clinically meaningful for patients based on the relatively small incremental benefits for OS and PFS.

A multicenter observational study (REGOTAS) (11) has recently demonstrated the clinical benefit and tolerability of these drugs in real-life clinical practice, and criteria to choose between regorafenib or TAS-102. However, the following issues remain to be established (12): The appropriate way of administration for patients with advanced disease, and which subpopulations of patients might derive the greatest benefit from salvage-line treatment with these drugs, compared to best supportive care only. To address these questions, we conducted a retrospective cohort study to evaluate the efficacy and safety of regorafenib and TAS-102 in patients with refractory mCRC with the aim of assessing their practical value as salvage-line therapy. A post-hoc exploratory subgroup analysis was carried out to obtain predictive scores for survival benefit in patients treated with these regimens.

\section{Patients and methods}

Patients. Patients with unresectable mCRC were eligible for the study if they had received at least two prior regimens of standard chemotherapies. All patients had been treated at Tokai University Hospital (Kanagawa, Japan) between June 2013 and November 2015, after the approval of each drug for medical reimbursement under the national insurance scheme in Japan (regorafenib and TAS-102 were approved in May 2013 and May 2014, respectively). The eligibility criteria were as follows: i) histologically confirmed adenocarcinoma of the colon or rectum, and presence of unresectable metastatic disease; ii) history of treatments with fluoropyrimidine, irinotecan, oxaliplatin, and anti-VEGF antibody (bevacizumab), or anti-epidermal growth factor receptor (EGFR) antibody (cetuximab or panitumumab) for patients who had KRAS exon 2 wild-type tumor; iii) Eastern Cooperative Oncology Group performance status (ECOG PS) of 0 to 2; and iv) adequate bone-marrow, liver, and renal function at the start of the treatment. Patients were excluded if they had previously received regorafenib or TAS-102, or had uncontrolled medical disorders.

The Institutional Review Board for Clinical Research approved all procedures for this retrospective observational study (no. 16R-190), which was conducted in accordance with the Declaration of Helsinki.

Treatment. Regorafenib (160 mg as a standard dose) was administered once daily on days $1-21$, with 7 days of rest. TAS-102 $\left(35 \mathrm{mg} / \mathrm{m}^{2}\right)$ was administered twice daily 5 days a week, with 2 days of rest, for 2 weeks, followed by a 14-day rest period. Both regimens were repeated every 4 weeks. The treatments were continued until disease progression, death, unacceptable toxicity, withdrawal of consent by the patient, or decision by the treating physician that discontinuation would be in the patient's best interest.

Patients whose initial dose had been reduced at the discretion of the treating physician were included in this study. Patients who required dose reductions could re-escalate the dose up to the recommended starting dose if the toxicity resolved to baseline level. All patients received the best supportive care available, but were not allowed to receive other antitumor agents, hormonal therapy, or immunotherapy. The decisions regarding which drug should be administered first, and whether to provide crossover between treatments, were made by the treating physicians (Fig. 1).

Evaluation. All patients underwent computed tomography every 8 weeks to assess tumor responses to therapy in terms of change from baseline during treatment according to the Response Evaluation Criteria in Solid Tumors (RECIST) version 1.1 (13). We defined PFS1 as the interval from the first administration of the primary treatment to the first radiologic or clinical observation of disease progression or death from any cause, whichever came first (Fig. 1).

We defined PFS2 as the interval from the initiation of the secondary treatment to the second progression, for those who had undertaken crossover between treatments after a first progression. We defined OS1 as the time between the administration date of the primary treatment and the date of death from any cause, and OS2 as the time between the administration date of the secondary treatment, if applicable, and the date of death. The median PFS1, PFS2, OS1 and OS2 were estimated using the Kaplan-Meier method.

The planned dose intensity (DI) for each drug was defined as the total amount of drug in the entire treatment intended based on the recommended dose and schedule. Then, the relative dose intensity (RDI) for each drug was calculated as the ratio between the delivered DI and the planned DI (14). Adverse events were classified and graded according to the National Cancer Institute Common Terminology Criteria for Adverse Events, version 4.03 (15).

Statistical methods and prognostic score construction. Parametric data with $\mathrm{P}>0.05$ for the Kolmogorov-Smirnov test were analyzed using Welch's two sample t-test, and non-parametric data using the Wilcoxon test. Categorical data were analyzed using Fisher's exact test. The PFS1, 2 and OS1, 2 were compared using a log-rank test with $95 \%$ confidence intervals (95\% CIs).

The results of OS1 were plotted against the total delivered dose or the RDI for each drug and fitted to a simple linear regression model to calculate the regression coefficient (16). A Cox proportional hazards regression model was used to test each candidate variable predictor associated with OS1 using stepwise model selection according to Akaike's information criterion. To take account of the small number of patients with PS 2, the values of ECOG PS were incorporated into the model as a numerical variable. Hazard ratios were calculated by taking the exponentials of the $\beta$ coefficients of Cox models. Model discrimination was done by calculating the Harrell's $C$ (for concordance) index, which is the area under the receiver operator curve $(17,18)$. Hazard ratios of covariates were rounded to the nearest integer to construct score weights. The range of possible total score weights was divided into three groups to stratify patients into poor-, intermediate- and long-survival tertiles. $\mathrm{P}<0.05$ was considered to indicate a statistically significant difference. All analyses were performed using $\mathrm{R}$ version 3.3.2 (The R Foundation for Statistical Computing Platform) (19).

\section{Results}

Patients. Patient demographics and characteristics are outlined in Table I. Between June 2013 and November 2015, 44 patients 


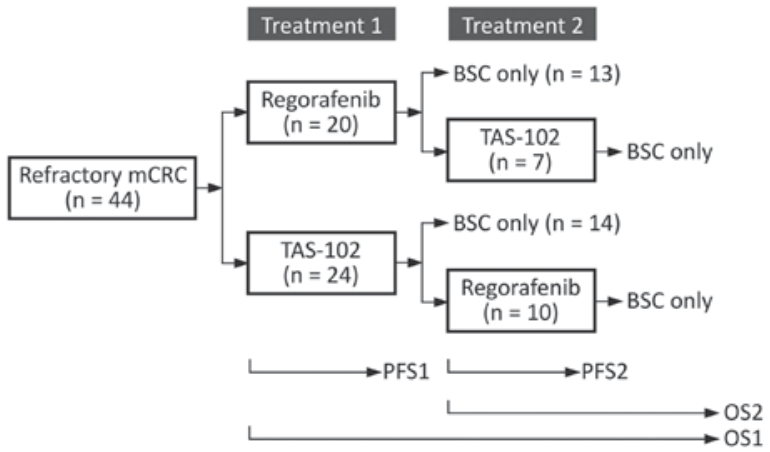

Figure 1. Flow diagram of salvage-line therapy. Each agent was administered at the discretion of the attending physician. mCRC, metastatic colorecta cancer; TAS-102, trifluridine and tipiracil; BSC, best supportive care; OS, overall survival; PFS, progression-free survival.

with mCRC who were treated with either regorafenib or TAS-102 for the first time were included in the analysis. Of these patients, 7 went on to receive TAS-102 and 10 went on to receive regorafenib as secondary treatment (Fig. 1). Baseline demographic and disease characteristics were well balanced between the two groups in terms of the primary treatment. All the patients had received prior chemotherapy regimens containing a fluoropyrimidine, oxaliplatin and irinotecan; all but one patient (in the group with primary use of TAS-102) had received bevacizumab.

Treatment exposure. Crossover between treatments was conducted for patients with ECOG PS 0 or 1 at the time when the first treatment was finished (Table II). The durations of treatment were not significantly different for regorafenib and TAS-102: median 2.6 months (range: 0.1-10.8) for regorafenib and 3.8 months (0.9-20.3) for TAS-102 in Treatment 1 , and then 4.2 months (0.4-12.9) and 3.7 months (0.9-15.1), respectively, in Treatment 2 . The starting dose rate of regorafenib was reduced to $0.78 \pm 0.26$ mean \pm standard deviation $(\mathrm{SD})$ in Treatment 1 , and to $0.71 \pm 0.10$ in Treatment 2 . Although the incidences of any dose modification were equivalent, the RDI over the whole treatment period was greater for TAS-102: $0.83 \pm 0.14$ for TAS-102 vs. $0.54 \pm 0.21$ for regorafenib in Treatment $1(\mathrm{P}<0.001)$, and $0.90 \pm 0.11$ vs. $0.63 \pm 0.16$ in Treatment $2(\mathrm{P}<0.001)$.

Efficacy. No patient had a complete response (CR) or partial response (PR), as shown in Table II. Disease control was achieved in 15 out of 20 patients $(75.0 \%)$ for regorafenib and 17 out of 24 patients $(70.8 \%$ ) for TAS-102 in Treatment 1 , and in 6 out of 10 patients $(60.0 \%)$ and 4 out of 7 patients $(57.1 \%)$ in Treatment 2, respectively. There was no difference in the best overall response for either treatment line.

Median OS1 was 9.1 months and $20 \%$ of patients were alive 12 months after starting regorafenib first; the corresponding values were 9.3 months and $25 \%$ for patients treated with TAS-102 first (Table II). As for secondary use, median OS2 values were 7.1 months and 5.3 months for regorafenib and TAS-102, respectively, and no patient was alive in either case at 12 months after crossover. There was no difference in outcomes between regorafenib and TAS-102, regardless of the order in which the two drugs were used.

There was clearly a relationship between OS1 and a total of delivered dose for each drug (Fig. 2A and B), even though
OS1 was not correlated to RDI (Fig. 2C and D). The correlation between OS1 and the total of delivered dose was higher for TAS-102, for which the data showed much less scatter, as shown in Fig. $2 \mathrm{~B}$.

Safety. Table III summarizes drug-related adverse events (AEs). Drug-related AEs occurred in 20 (100\%) patients for regorafenib and in $22(92 \%)$ patients for TAS-102 in Treatment 1 , and then in $8(80 \%)$ and $7(100 \%)$, respectively, after crossover between the drugs in Treatment 2 . The frequencies of grade 3 or 4 hand-foot skin reaction (HFSR), increased aspartate transaminase, increased alanine transaminase, and increased bilirubin for the secondary use of regorafenib after TAS-102 were not greater than the frequencies for the primary use of regorafenib (10\% vs. $45 \%, 0 \%$ vs. $5 \%, 0 \%$ vs. $5 \%, 0 \%$ vs. $10 \%$, respectively). Similarly, the frequencies of grade 3 or 4 leukopenia, neutropenia, anemia, and nausea for the secondary use of TAS-102 posterior to regorafenib were similar to those for the primary use of TAS-102 (57\% vs. $29 \%, 29 \%$ vs. $34 \%$, $57 \%$ vs. $38 \%, 0 \%$ vs. $8 \%$, respectively). One patient during the primary administration of regorafenib suffered from severe treatment-related liver dysfunction, and discontinued the treatment after recovery.

Prognostic score. Table IV summarizes the findings of univariate and multivariate analyses of baseline characteristics as prognostic factors for OS and the score weights assigned to each retained predictor variable. The total possible score was 12 points; however, no patient had a score $>10$. Patients who had a worse ECOG PS, time since diagnosis of metastatic disease $\leq 18$ months (rapid growth of tumor), and prior chemotherapy continued $\geq 2$ months beyond progressive disease (PD) on the RECIST criteria (including so-called clinical PD) showed a $\mathrm{P}$-value $<0.05$ in the univariate analysis examining the association between baseline characteristics and poor OS.

Prior chemotherapy was repeated every 2 to 3 weeks according to a regimen with an evaluation interval of $<3$ months, so regorafenib or TAS-102 could be started within 6 weeks after failure of the prior chemotherapy. Continuation of prior chemotherapy $\geq 2$ months beyond PD would represent prolonged administration that clinicians intended to conduct for some reason.

In multivariate analysis, these three factors remained significant for the parsimonious model to predict OS while retaining good discrimination $(C$-statistic $=0.70)$. A score of 0-3 defined long survival; $4-5$, intermediate survival; and $\geq 6$, poor survival (Fig. 3).

\section{Discussion}

Regorafenib and TAS-102 have been reported to show similar efficacy but different toxicity profiles for regorafeniband TAS-102-naive patients in retrospective cohort studies $(11,20,21)$. Analyses of efficacy and safety in patients treated with regorafenib or TAS-102 in the real-life setting are important for clinicians, because patient characteristics in real-life, especially ECOG PS, may differ from those in phase III trials (7-9).

No patient had a CR or PR for either drug in our cohort. The two drugs were equivalent in terms of DCR: $75.0 \%$ for 
Table I. Demographics.

Primary treatment

Characteristic

Regorafenib (n=20)

TAS-102 $(n=24)$

P-value

Age, median [range]

68 [57-78]

64 [44-86]

0.087

Sex

Male

$13(65.0)$

$15(62.5)$

Female

7 (35.0)

9 (37.5)

ECOG PS

0

$6(30.0)$

$14(58.3)$

0.077

1

$12(60.0)$

$6(25.0)$

2 (10.0)

4 (16.7)

Primary site of disease

Right colon

4 (20.0)

$10(41.7)$

7 (35.0)

3 (12.5)

Left colon

9 (37.5)

$11(45.8)$

KRAS exon 2 status

Wild

$9(45.0)$

$14(58.3)$

$11(55.0)$

$10(41.7)$

Number of prior regimens

2

$12(60.0)$

$12(50.0)$

0.87

3

$8(40.0)$

$11(45.8)$

$\geq 4$

0 (0)

1 (4.2)

Number of metastatic sites

$$
1
$$

$6(30.0)$

$6(25.0)$

0.46

$12(60.0)$

$11(45.8)$

$>3$

2 (10.0)

7 (29.2)

Metastatic site

Liver

$16(80.0)$

$19(79.2)$

0.26

Lung

$10(50.0)$

$13(54.2)$

$6(30.0)$

4 (16.7)

Peritoneum

$2(10.0)$

$8(33.3)$

Others

$2(10.0)$

$8(33.3)$

Time from initiation of first-line chemotherapy

$$
\begin{aligned}
& \leq 18 \text { months } \\
& >18 \text { months }
\end{aligned}
$$

$6(25.0)$

$15(75.0)$

$18(75.0)$

History of systemic anticancer agents

Fluoropyrimidine

20 (100)

24 (100)

24 (100)

Oxaliplatin

20 (100)

$24(100)$

Irinotecan

20 (100)

$23(95.8)$

$20(100)$

$11(45.8)$

Anti-EGFR antibody (Wild KRAS or all-RAS $S^{\mathrm{a}}$

Post-treatment use of regorafenib or TAS-102

$9(45.0)$

$10(41.7)$

Non-parametric data with $\mathrm{P}<0.05$ for Kolmogorov-Smirnov test are presented as the median [range] and were examined using the Wilcoxon rank sum test. Categorical data are accompanied by percentage in parentheses and were examined using Fisher's exact test. ${ }^{a}$ An all-RAS test was approved in Japan in April, 2015. Anti-EGFR antibody was subsequently applied based on all-RAS test. ECOG PS, Eastern Cooperative Oncology Group performance status; TAS-102, trifluridine and tipiracil; KRAS, Kirsten rat sarcoma viral oncogene homolog VEGF, vascular endothelial growth factor; EGFR, epidermal growth factor receptor.

regorafenib and $70.8 \%$ for TAS-102 in primary salvage treatment, and 60.0 and $57.1 \%$, respectively, in secondary use.
Crossover administration was achieved in 7 out of $20(35.0 \%)$ patients treated with regorafenib first, and in 10 out of $24(41.7 \%)$ 
Table II. Administration of study drugs, response and survival.

A, Treatment 1 (primary use).

\begin{tabular}{|c|c|c|c|}
\hline Variable & $\begin{array}{c}\text { Regorafenib } \\
n=20\end{array}$ & $\begin{array}{c}\text { TAS-102 } \\
n=24\end{array}$ & P-value \\
\hline \multicolumn{4}{|l|}{ ECOG PS, n (\%) } \\
\hline 0 & $6(30.0)$ & $14(58.3)$ & \multirow[t]{3}{*}{0.077} \\
\hline 1 & $12(60.0)$ & $6(25.0)$ & \\
\hline 2 & $2(10.0)$ & $4(16.7)$ & \\
\hline Median period of medication, months & 2.6 [range: $0.1-10.8$ ] & $3.8[0.9-20.3]$ & 0.18 \\
\hline Relative initial dose, mean \pm SD & $0.78 \pm 0.26$ & $0.97 \pm 0.09$ & 0.0031 \\
\hline Any treatment modification, $\mathrm{n}(\%)$ & $19(95.0)$ & $18(75.0)$ & 0.11 \\
\hline Mean RDI \pm SD & $0.54 \pm 0.21$ & $0.83 \pm 0.14$ & $<0.001$ \\
\hline Median OS1, months & 9.1 (95\% CI: 4.1-13.4) & $9.3(5.5-12.3)$ & 0.68 \\
\hline Patients alive at 12 months, $\mathrm{n}(\%)$ & $4(20.0)$ & $6(25.0)$ & 0.73 \\
\hline Median PFS1, months & 2.1 (95\% CI: $1.3-3.6)$ & $3.1(1.7-4.1)$ & 0.13 \\
\hline \multicolumn{4}{|l|}{ Best overall response $^{\mathrm{a}}, \mathrm{n}(\%)$} \\
\hline $\mathrm{CR}$ & $0(0)$ & $0(0)$ & \multirow[t]{4}{*}{1.0} \\
\hline PR & $0(0)$ & $0(0)$ & \\
\hline SD & $15(75.0)$ & $17(70.8)$ & \\
\hline PD & $5(25.0)$ & 7 (29.2) & \\
\hline
\end{tabular}

B, Treatment 2 (secondary use).

\begin{tabular}{|c|c|c|c|}
\hline Variable & $\begin{array}{l}\text { Regorafenib } \\
n=10\end{array}$ & $\begin{array}{c}\text { TAS-102 } \\
n=7\end{array}$ & P-value \\
\hline \multicolumn{4}{|l|}{ ECOG PS, n (\%) } \\
\hline 0 & $1(10.0)$ & $3(42.9)$ & \multirow[t]{3}{*}{0.12} \\
\hline 1 & $9(90.0)$ & $4(57.1)$ & \\
\hline 2 & $0(0)$ & $0(0)$ & \\
\hline Median period of medication, months & 4.2 [range: $0.4-12.9$ ] & $3.7[0.9-15.1]$ & 0.80 \\
\hline Relative initial dose, mean \pm SD & $0.71 \pm 0.10$ & $0.94 \pm 0.15$ & 0.0058 \\
\hline Any treatment modification, $\mathrm{n}(\%)$ & $10(100)$ & $4(57.1)$ & 0.051 \\
\hline Mean RDI \pm SD & $0.63 \pm 0.16$ & $0.90 \pm 0.11$ & $<0.001$ \\
\hline Median OS2, months & 7.1 (95\% CI: 5.0-NA) & $5.3(3.0-N A)$ & 0.67 \\
\hline Patients alive at 12 months, n (\%) & $0(0)$ & $0(0)$ & 1.0 \\
\hline Median PFS2, months & 3.7 (95\% CI: 3.1-NA) & $3.7(0.8-N A)$ & 0.23 \\
\hline \multicolumn{4}{|l|}{ Best overall response $^{\mathrm{a}}, \mathrm{n}(\%)$} \\
\hline $\mathrm{CR}$ & $0(0)$ & $0(0)$ & \multirow[t]{4}{*}{1.0} \\
\hline PR & $0(0)$ & $0(0)$ & \\
\hline $\mathrm{SD}$ & $6(60.0)$ & $4(57.1)$ & \\
\hline $\mathrm{PD}$ & $4(40.0)$ & $3(42.9)$ & \\
\hline
\end{tabular}

${ }^{a}$ Response evaluation criteria in solid tumors (RECIST) version 1.1; ECOG PS, Eastern Cooperative Oncology Group performance status; $\pm \mathrm{SD}$, standard deviation; RDI, relative dose intensity; OS, overall survival; PFS, progression-free survival; CI, confidence interval; $N A$, not available; $\mathrm{CR}$, complete response; $\mathrm{PR}$, partial response; $\mathrm{SD}$, stable disease; $\mathrm{PD}$, progressive disease.

patients treated with TAS-102 first, but this does not imply inferiority: We found that TAS-102 had provided a prolonged period of medication for patients with poor performance status (ECOG PS=2), as shown in Table II. Median OS1 of 4 patients with ECOG PS=2 at the time of study entry was 3 months (range: 1.3 to 5.5 months) for TAS-102, providing 
Table III. Adverse events.

\begin{tabular}{|c|c|c|c|c|c|c|c|c|c|c|}
\hline \multirow[b]{3}{*}{$\mathrm{n}(\%)$} & \multicolumn{4}{|c|}{ Regorafenib (n=30) } & \multirow[b]{3}{*}{$\mathrm{P}$-value ${ }^{\mathrm{a}}$} & \multicolumn{4}{|c|}{ TAS-102 (n=31) } & \multirow[b]{3}{*}{ P-value ${ }^{a}$} \\
\hline & \multicolumn{2}{|c|}{$\begin{array}{c}\text { REG-only or } \\
\text { REG prior to TAS } \\
(\mathrm{n}=20)\end{array}$} & \multicolumn{2}{|c|}{$\begin{array}{l}\text { REG posterior } \\
\text { to TAS } \\
(\mathrm{n}=10)\end{array}$} & & \multicolumn{2}{|c|}{$\begin{array}{l}\text { TAS-only or } \\
\text { TAS prior to } \\
\text { REG }(n=24)\end{array}$} & \multicolumn{2}{|c|}{$\begin{array}{l}\text { TAS posterior } \\
\text { to REG } \\
\quad(n=7)\end{array}$} & \\
\hline & $\begin{array}{l}\text { Any } \\
\text { grade }\end{array}$ & $\geq$ Grade 3 & $\begin{array}{l}\text { Any } \\
\text { grade }\end{array}$ & $\geq$ Grade 3 & & $\begin{array}{l}\text { Any } \\
\text { grade }\end{array}$ & $\geq$ Grade 3 & $\begin{array}{l}\text { Any } \\
\text { grade }\end{array}$ & $\geq$ Grade 3 & \\
\hline Any event & $20(100)$ & $13(65)$ & $8(80)$ & $5(50)$ & 0.69 & $22(92)$ & $15(63)$ & $7(100)$ & $6(86)$ & 0.20 \\
\hline \multicolumn{11}{|l|}{ Clinical AEs } \\
\hline HFSR & $14(70)$ & $9(45)$ & $3(30)$ & $1(10)$ & 0.077 & $0(0)$ & $0(0)$ & $0(0)$ & $0(0)$ & $N A$ \\
\hline Nausea & $5(25)$ & $0(0)$ & $0(0)$ & $0(0)$ & 0.038 & $10(42)$ & $2(8)$ & $4(57)$ & $0(0)$ & 0.96 \\
\hline Anorexia & $9(45)$ & $1(5)$ & $1(10)$ & $0(0)$ & 0.33 & $8(36)$ & $2(9)$ & $4(57)$ & $0(0)$ & 1.0 \\
\hline Diarrhea & $2(10)$ & $0(0)$ & $1(10)$ & $0(0)$ & 0.090 & $3(13)$ & $0(0)$ & $1(14)$ & $0(0)$ & 0.021 \\
\hline Fatigue & $11(55)$ & $2(10)$ & $4(40)$ & $0(0)$ & 0.31 & $12(50)$ & $1(4)$ & $3(43)$ & $0(0)$ & 0.87 \\
\hline Mucositis oral & $4(20)$ & $0(0)$ & $0(0)$ & $0(0)$ & 0.30 & $6(25)$ & $1(4)$ & $1(14)$ & $0(0)$ & 0.37 \\
\hline Hypertension & $9(45)$ & $2(10)$ & $2(20)$ & $2(20)$ & 0.33 & $0(0)$ & $0(0)$ & $0(0)$ & $0(0)$ & $N A$ \\
\hline Voice alteration & $6(30)$ & $0(0)$ & $0(0)$ & $0(0)$ & 0.098 & $0(0)$ & $0(0)$ & $0(0)$ & $0(0)$ & $N A$ \\
\hline Alopecia & $0(0)$ & $0(0)$ & $0(0)$ & $0(0)$ & $N A$ & $1(4)$ & $0(0)$ & $2(29)$ & $0(0)$ & 0.13 \\
\hline Others & $0(0)$ & $0(0)$ & $3(29)$ & $3^{\mathrm{b}}(30)$ & 0.038 & $0(0)$ & $0(0)$ & $0(0)$ & $0(0)$ & $N A$ \\
\hline \multicolumn{11}{|l|}{ Laboratory abnormalities } \\
\hline Leukopenia & $0(0)$ & $0(0)$ & $0(0)$ & $0(0)$ & 1.0 & $12(50)$ & $7(29)$ & $4(57)$ & $4(57)$ & 0.70 \\
\hline Neutropenia & $0(0)$ & $0(0)$ & $0(0)$ & $0(0)$ & 1.0 & $13(54)$ & $9(34)$ & $3(43)$ & $2(29)$ & 0.92 \\
\hline Anemia & $0(0)$ & $0(0)$ & $2(20)$ & $0(0)$ & 0.15 & $15(63)$ & $9(38)$ & $5(71)$ & $4(57)$ & 0.89 \\
\hline Thrombocytopenia & $6(30)$ & $2(10)$ & $2(20)$ & $1(10)$ & 0.96 & $7(29)$ & $2(8)$ & $2(29)$ & $0(0)$ & 0.96 \\
\hline AST increased & $15(75)$ & $1(5)$ & $4(40)$ & $0(0)$ & 0.26 & $6(25)$ & $1(4)$ & $4(57)$ & $2(29)$ & 0.44 \\
\hline ALT increased & $6(30)$ & $1(5)$ & $2(29)$ & $0(0)$ & 0.40 & $4(17)$ & $0(0)$ & $5(71)$ & $1(14)$ & 0.020 \\
\hline Hyperbilirubinemia & $7(35)$ & $2(10)$ & $1(10)$ & $0(0)$ & 0.61 & $1(4)$ & $0(0)$ & $3(43)$ & $1(14)$ & 0.030 \\
\hline Discontinuation due to AEs & \multicolumn{2}{|c|}{$5(25)$} & \multicolumn{2}{|c|}{$2(20)$} & 0.75 & \multicolumn{2}{|c|}{$3(13)$} & \multicolumn{2}{|c|}{$0(0)$} & 0.78 \\
\hline
\end{tabular}

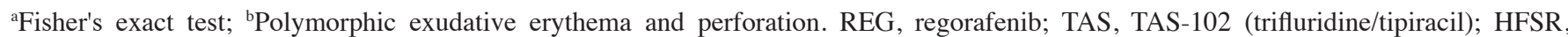
hand-foot skin reaction; AE, adverse event; NA, not applicable; AST, aspartate aminotransferase; ALT, alanine aminotransferase. Categorical data are accompanied by percentages in parentheses.

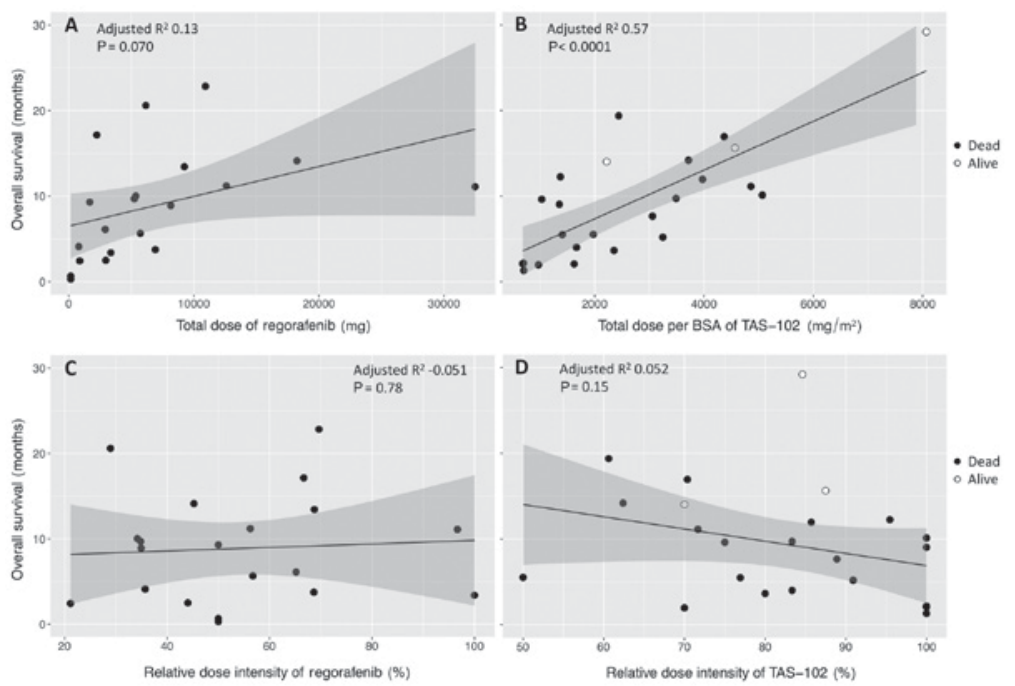

Figure 2. Effect of delivered dose on survival time, the time between the administration date of the primary treatment and the date of death from any cause (OS1). OS1 is plotted against total dose of (A) regorafenib or (B) TAS-102, and RDI of (C) regorafenib or (D) TAS-102. Among the patients with primary TAS-102, three (indicated by open circles) were alive at the time of data collection. The regression line is drawn with the $95 \%$ confidence intervals (gray shadows). BSA, body surface area; RDI, relative dose intensity; TAS-102, trifluridine/tipiracil. 
Table IV. Predictors for OS1 in patients treated with regorafenib and/or TAS-102.

\begin{tabular}{|c|c|c|c|c|c|}
\hline \multirow[b]{2}{*}{ Characteristic } & \multicolumn{2}{|c|}{ Univariate model } & \multicolumn{2}{|c|}{ Multivariate model } & \multirow[b]{2}{*}{ Score weight } \\
\hline & HR $(95 \%$ CI) & P-value & HR (95\% CI) & P-value & \\
\hline Male sex & $1.85(0.912-3.75)$ & 0.089 & & & \\
\hline Age & $0.992(0.960-1.03)$ & 0.62 & & & \\
\hline ECOG PS & $1.78(1.02-3.10)$ & 0.044 & $2.00(1.13-3.53)$ & 0.018 & 2 \\
\hline \multicolumn{6}{|l|}{ Primary lesion } \\
\hline Right colon & $1.53(0.631-3.73)$ & 0.35 & & & \\
\hline Rectum & $1.49(0.642-3.47)$ & 0.35 & & & \\
\hline \multicolumn{6}{|l|}{$K R A S$ exon 2 status } \\
\hline Mutant & $0.985(0.526-1.84)$ & 0.96 & & & \\
\hline \multicolumn{6}{|l|}{ Metastatic sites } \\
\hline $\mathrm{n} \geq 3$ & $1.21(0.550-2.65)$ & 0.64 & & & \\
\hline \multicolumn{6}{|l|}{ Metastatic sites } \\
\hline Liver & $1.18(0.538-2.58)$ & 0.68 & & & \\
\hline Lung & $1.28(0.688-2.40)$ & 0.43 & & & \\
\hline Peritoneum & $1.01(0.477-2.12)$ & 0.99 & & & \\
\hline Lymph node & $0.596(0.271-1.31)$ & 0.20 & & & \\
\hline Other & $1.17(0.570-2.41)$ & 0.67 & & & \\
\hline Number of prior regimens & $0.876(0.528-1.46)$ & 0.61 & & & \\
\hline \multicolumn{6}{|l|}{ History of biologicals } \\
\hline Anti-VEGF antibody & $1.13(0.153-8.30)$ & 0.91 & & & \\
\hline Anti-EGFR antibody & $0.827(0.438-1.56)$ & 0.56 & & & \\
\hline \multicolumn{6}{|c|}{ Time since diagnosis of metastatic disease } \\
\hline$\leq 18$ months & $2.17(1.04-4.55)$ & 0.039 & $2.51(1.17-5.37)$ & 0.018 & 3 \\
\hline \multicolumn{6}{|l|}{ Prior chemotherapy } \\
\hline Continued $\geq 2$ months beyond PD & $3.62(1.72-7.63)$ & $<0.001$ & $4.95(2.20-11.1)$ & $<0.001$ & 5 \\
\hline Harrell's C-index & & & 0.70 & & \\
\hline Total possible score & & & & & 12 \\
\hline
\end{tabular}

Referent primary lesion is the left colon, referent number of metastatic sites is 1 to 2 , and referent prior chemotherapy is discontinued at the time of diagnosis of PD, or for other reasons (unacceptable adverse events, or increase in serum level of carcinoembryonic antigen). OS1, the time between the administration date of the primary treatment and the date of death from any cause; HR, hazard ratio; CI, confidence interval; ECOG PS, Eastern Cooperative Oncology Group performance status; VEGF, vascular endothelial growth factor; EGFR, epidermal growth factor receptor; $\mathrm{PD}$, progressive disease.

better survival compared to two weeks for 2 patients treated with regorafenib $(\mathrm{P}=0.020)$.

A cohort study of regorafenib in real-life clinical practice for mCRC patients in France (REBECCA) (22) found that $50 \%$ of patients had a treatment modification (dose reduction or interruption), and $31 \%$ of patients discontinued regorafenib before progression mainly due to toxicity or deterioration of general health status. According to their data, survival was unfavorably affected by a low initial daily dose of regorafenib.

Median OS1 in our study was consistent with those in CORRECT (7) (median OS, 6.4 months; 12-month survival, 24\%) and REBECCA (22) (5.6 months; 22\%) for regorafenib, and RECOURSE (9) (7.1 months; 27\%) for TAS-102, although 95 or $75 \%$ of our patients treated with regorafenib or TAS-102 first, respectively, had a dose modification (Table II). An initial dose was more likely to be reduced for regorafenib compared to TAS-102 to avoid early AE within the first 3 weeks of regorafenib treatment, but there was no correlation between deterioration of OS1 and reduction of initial daily dose (data not shown). This may be because we commonly escalated the dosage thereafter, if possible, up to $120 \mathrm{mg}$ (4 out of 11 patients) or $160 \mathrm{mg}$ (1 out of 10 patients), based on each patient's response. The most common dosage was $120 \mathrm{mg}$ daily (19 out of 30 patients) in the first 2 cycles, as recently recommended in the ReDOS study (23). It seemed important for successful escalation of regorafenib to inform patients before administration about the likelihood of weekly dose escalation. Furthermore, OS1 for regorafenib or TAS-102, regardless of single use or crossover, was not correlated to RDI, but was proportional to a total dose of each drug (Fig. 2). In third-line or later treatments, clinicians may continue to prescribe the maximum recommended dose to obtain the best outcome, but may withdraw treatment from patients whose performance status deteriorates. In the former scenario, patients could experience adverse effects without any benefit, whereas possible responders 
could be missed in the latter scenario. Our results indicate that lower dose-intensity provides a longer duration of life under treatment compared to higher dose-intensity in some cases. This can be interpreted as indicating that there was a greater improvement in survival when these drugs were administered at the appropriate dose for each individual patient and continued for as long as possible until progression. Regarding regorafenib, Osawa (24) recommended an initial dose of $120 \mathrm{mg}$ for salvage treatment of $\mathrm{mCRC}$, as this provided a significant effect with good tolerability.

It has been considered that the toxic effects of TAS-102 are generally mild and manageable compared with those of regorafenib (21). The reported incidence of clinical AEs for regorafenib, including grade $\geq 3$ HFSR (17\% of patients in CORRECT) (7), fatigue (10\%), and hepatotoxicity $(6 \%$ of Asian population in CONCUR) (8), makes it difficult to administer regorafenib to patients who have previously been treated with TAS-102. In this study, the safety profiles of regorafenib and TAS-102 were broadly consistent with those in previous pivotal trials $(7-9,25)$ (Table III). In addition, the incidences of HFSR, fatigue and hepatotoxicity in patients given regorafenib were not significantly increased even if the drug was used after TAS-102, while conversely, the frequencies of myelosuppression including leukopenia and neutropenia, nausea and anorexia in patients given TAS-102 were not greater in patients with previous regorafenib treatment. Although treatment discontinuation due to toxic effects was more frequently observed for regorafenib treatment, the incidence of toxic effects was not increased in patients with previous TAS-102 treatment, provided that the initial dose of regorafenib was reduced to $120 \mathrm{mg}$ in most cases (Table II). These results indicate that regorafenib can be safely administered to patients with previous TAS-102 treatment.

Predictive biomarkers for OS have not yet been identified for $\mathrm{mCRC}$ patients treated with regorafenib or TAS-102 $(11,26)$. No association was identified between $K R A S, B R A F$ and PIK3CA mutation status and outcomes in CORRECT (7) and RECOURSE (9). The post hoc analysis of CORRECT indicated that patients treated with regorafenib who had long PFS ( $>4$ months) tended to have a better ECOG PS (score, 0), fewer metastatic tumor sites (1 to 2 sites), and a longer time ( $\geq 18$ months) since diagnosis of metastatic disease (25). In contrast, REBECCA (22) indicated that the following 6 baseline variables were associated with poorer survival: poor ECOG PS, a shorter time from diagnosis of metastases, a low initial dose of regorafenib, $>3$ metastatic sites, liver metastases, and KRAS mutations. A longer time since diagnosis of metastatic disease is considered to reflect a better response to chemotherapy, so PFS2 after success of crossover between regorafenib and TAS-102 tended to be longer than PFS1, which included patients with rapidly growing tumors refractory to treatments (Table II).

In our study, a model with a good discrimination $(C$-index 0.70 ), consisting of only 3 baseline predictors (poor ECOG PS, $\leq 18$ months from diagnosis of metastases, and prior chemotherapy continued $\geq 2$ months beyond PD) (Table IV), classified patients into similar prognostic groups (Fig. 3). We recommend that patients having a high probability of benefit should be identified before starting treatment with regorafenib or TAS-102 among patients refractory to standard chemotherapy.

It remains an important clinical issue to decide which drug should be administered first, but this has not been established

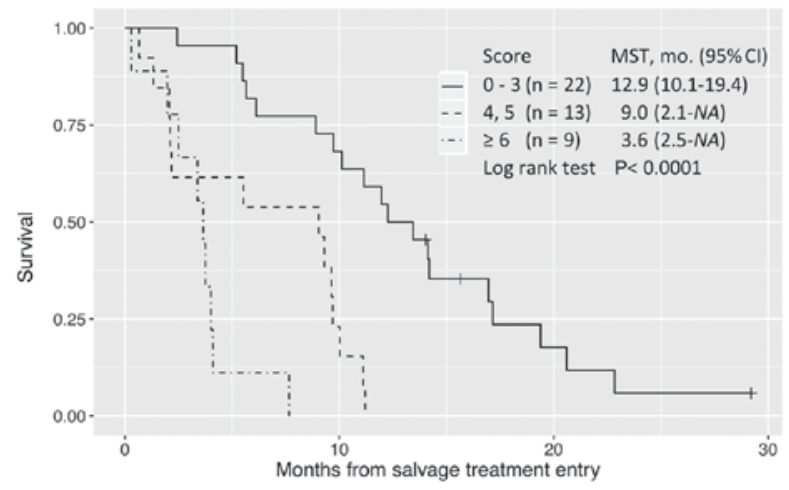

Figure 3. Overall survival since study entry (OS1) stratified by the prognostic score. Patients with a score of $\leq 3$ exhibited relatively prolonged lifetimes. MST, mean survival time; CI, confidence interval.

because of the lack of a head-to-head randomized trial. A retrospective comparative analysis in 550 patients (REGOTAS) (11) suggested that regorafenib should be given first in patients aged $<65$ years, but TAS- 102 in patients aged $\geq 65$ years, based on a favorable trend of OS. We tried a propensity score method (inverse probability of treatment weighting) (27) for choosing between the two drugs in our study, but there was no clear result, except for a favorable trend with age in the TAS-102-first group (hazard ratio, 0.8; 95\% confidence interval, 0.7-0.9). No difference in OS was found between the two drugs.

There are some potential limitations of this study. Our study is a retrospective single-center analysis. Although all patients with refractory mCRC treated with regorafenib or TAS-102 in the period were included, the number of patients was relatively small. All patients were treated by a team of six surgeons, all of whom are colorectal cancer specialists. An all-RAS (KRAS and $N R A S$ ) test was approved in Japan on April, 2015. Among our patients, 15 had wild-KRAS exon 2 tumor identified before that date, and all of them received anti-EGFR antibody regimens. Although $20 \%$ of them (3 patients) might have other RAS mutation, the number is too small to permit any conclusion; at worst, they would have had a short duration of anti-EGFR treatment without benefit until tumor progression. External validation is still needed to confirm the model used to predict OS.

This analysis suggests that the administration of regorafenib and TAS-102 can be recommended for patients with refractory mCRC who have a better performance status, and a longer time since diagnosis of metastatic disease. Prolongation of the previous chemotherapy after diagnosis of disease progression attenuated the survival benefit of regorafenib and TAS-102, regardless of the order of their administration. We suggest that the optimal survival benefit of regorafenib and TAS-102 is provided by flexible and careful titration to the optimal dose for each individual patient with initial dose reduction if necessary, followed by prolonged administration until disease progression.

\section{Acknowledgements}

Not applicable.

\section{Funding}

No funding was received. 


\section{Availability of data and materials}

The datasets used and/or analyzed during the current study are available from the corresponding author on reasonable request.

\section{Authors' contributions}

Conception, design, collection of patient information, data interpretation, and drafting of the article was undertaken by AT. SS, TS, KO, GS and HM participated in patient treatment, and helped revise the manuscript. All authors read and approved the final manuscript.

\section{Ethics approval and consent to participate}

The Institutional Review Board for Clinical Research approved all procedures for this retrospective observational study (no. 16R-190), which was conducted in accordance with the Declaration of Helsinki.

\section{Patient consent for publication}

Not applicable.

\section{Competing interests}

The authors declare that they have no competing interests.

\section{References}

1. Howlader N, Noone A, Krapcho M, Garshell J, Miller D, Altekruse SF, Kosary CL, Yu M, Ruhl J, Tatalovich Z, et al: SEER Cancer Statistics Review, 1975-2012. National Cancer Institute, Bethesda, MD, USA, 2015.

2. Abrams TA, Meyer G, Schrag D, Meyerhardt JA, Moloney J and Fuchs CS: Chemotherapy usage patterns in a US-wide cohort of patients with metastatic colorectal cancer. J Natl Cancer Inst 106 djt371, 2014.

3. Wilhelm SM, Dumas J, Adnane L, Lynch M, Carter CA, Schütz G, Thierauch KH and Zopf D: Regorafenib (BAY 73-4506): A new oral multikinase inhibitor of angiogenic, stromal and oncogenic receptor tyrosine kinases with potent preclinical antitumor activity. Int J Cancer 129: 245-255, 2011.

4. Lenz HJ, Stintzing S and Loupakis F: TAS-102, a novel antitumor agent: A review of the mechanism of action. Cancer Treat Rev 41: 777-783, 2015.

5. Crawford J, Becker PS, Armitage JO, Blayney DW, Chavez J, Curtin P, Dinner S, Fynan T, Gojo I, Griffiths EA, et al: Myeloid growth factors, version 2.2017, NCCN clinical practice guidelines in oncology. J Natl Compr Canc Netw 15: 1520-1541, 2017.

6. Van Cutsem E, Cervantes A, Adam R, Sobrero A, Van Krieken JH, Aderka D, Aranda Aguilar E, Bardelli A, Benson A, Bodoky G, et al: ESMO consensus guidelines for the management of patients with metastatic colorectal cancer. Ann Oncol 27: 1386-1422, 2016.

7. Grothey A, Van Cutsem E, Sobrero A, Siena S, Falcone A, Ychou M, Humblet Y, Bouché O, Mineur L, Barone C, et al: Regorafenib monotherapy for previously treated metastatic colorectal cancer (CORRECT): An international, multicentre, randomised, placebo-controlled, phase 3 trial. Lancet 381: 303-312, 2013.

8. Li J, Qin S, Xu R, Yau TC, Ma B, Pan H, Xu J, Bai Y, Chi Y, Wang L, et al: Regorafenib plus best supportive care versus placebo plus best supportive care in Asian patients with previously treated metastatic colorectal cancer (CONCUR): A randomised, double-blind, placebo-controlled, phase 3 trial. Lancet Oncol 16: 619-629, 2015.

9. MayerRJ,Van Cutsem E,Falcone A, Yoshino T, Garcia-Carbonero R, Mizunuma N, Yamazaki K, Shimada Y, Tabernero J, Komatsu Y, et al: Randomized trial of TAS-102 for refractory metastatic colorectal cancer. N Engl J Med 372: 1909-1919, 2015.
10. Xu J, Kim TW, Shen L, Sriuranpong V, Pan H, Xu R, Guo W, Han SW, Liu T, Park YS, et al: Results of a randomized, double-blind, placebo-controlled, phase III trial of trifluridine/tipiracil (TAS-102) monotherapy in Asian patients with previously treated metastatic colorectal cancer: The TERRA study. J Clin Oncol 36: 350-358, 2018.

11. Moriwaki T, Fukuoka S, Taniguchi H, Takashima A, Kumekawa Y, Kajiwara T, Yamazaki K, Esaki T, Makiyama C, Denda T, et al: Propensity score analysis of regorafenib versus trifluridine/tipiracil in patients with metastatic colorectal cancer refractory to standard chemotherapy (REGOTAS): A Japanese society for cancer of the colon and rectum multicenter observational study. Oncologist 23: 7-15, 2018.

12. García-Alfonso P, Feliú J, García-Carbonero R, Grávalos C, Guillén-Ponce C, Sastre J and García-Foncillas J: Is regorafenib providing clinically meaningful benefits to pretreated patients with metastatic colorectal cancer? Clin Transl Oncol 18: 1072-1081, 2016

13. Eisenhauer EA, Therasse P, Bogaerts J, Schwartz LH, Sargent D, Ford R, Dancey J, Arbuck S, Gwyther S, Mooney M, et al: New response evaluation criteria in solid tumours: Revised RECIST guideline (version 1.1). Eur J Cancer 45: 228-247, 2009.

14. Hryniuk W and Bush $\mathrm{H}$ : The importance of dose intensity in chemotherapy of metastatic breast cancer. J Clin Oncol 2: 1281-1288, 1984.

15. Health and Human Services, National Institutes of Health and National Cancer Institute: Common terminology criteria for adverse events (CTCAE) v4.03, 2013. https://www.eortc.be/services/doc/ctc/ CTCAE_4.03_2010-06-14_QuickReference_5x7.pdf. Accessed: August $2 \overline{0}, 201 \overline{3}$

16. Wilkinson GN and Rogers CE: Symbolic description of factorial models for analysis of variance. Appl Stat-J R Stat Soc 22: 392-399, 1973.

17. Harrell FE Jr, Lee KL and Mark DB: Multivariable prognostic models: Issues in developing models, evaluating assumptions and adequacy, and measuring and reducing errors. Stat Med 15: 361-387, 1996.

18. Pencina MJ and D'Agostino RB: Overall C as a measure of discrimination in survival analysis: Model specific population value and confidence interval estimation. Stat Med 23: 2109-2123, 2004.

19. R Development Core Team: R: A language and environment for statistical computing. R Foundation for Statistical Computing, Vienna, Austria, 2016.

20. Masuishi T, Taniguchi H, Hamauchi S, Komori A, Kito Y, Narita Y, Tsushima T, Ishihara M, Todaka A, Tanaka T, et al: Regorafenib versus trifluridine/tipiracil for refractory metastatic colorectal cancer: A retrospective comparison. Clin Colorectal Cancer 16: e15-e22, 2017.

21. Sueda T, Sakai D, Kudo T, Sugiura T, Takahashi H, Haraguchi N, Nishimura J, Hata T, Hayashi T, Mizushima T, et al: Efficacy and safety of regorafenib or TAS-102 in patients with metastatic colorectal cancer refractory to standard therapies. Anticancer Res 36: 4299-4306, 2016.

22. Adenis A, de la Fouchardiere C, Paule B, Burtin P, Tougeron D, Wallet J, Dourthe LM, Etienne PL, Mineur L, Clisant S, et al: Survival, safety, and prognostic factors for outcome with Regorafenib in patients with metastatic colorectal cancer refractory to standard therapies: Results from a multicenter study (REBECCA) nested within a compassionate use program. BMC Cancer 16: 412, 2016.

23. Bekaii-Saab TS, Ou FS, Anderson DM, Ahn DH, Boland PM, Ciombor KK, Jacobs NL, Desnoyers RJ, Cleary JM, Meyers JP, et al: Regorafenib dose optimization study (ReDOS): Randomized phase II trial to evaluate dosing strategies for regorafenib in refractory metastatic colorectal cancer (mCRC)- An ACCRU network study. J Clin Oncol 36 (4 Suppl): S611, 2018.

24. Osawa H: Response to regorafenib at an initial dose of $120 \mathrm{mg}$ as salvage therapy for metastatic colorectal cancer. Mol Clin Oncol 6: 365-372, 2017.

25. Grothey A, Falcone A, Humblet Y, Bouche O, Mineur L, Adenis A, Tabernero J, Yoshino T, Lenz HJ and Goldberg RM: Characteristics of patients (pts) with metastatic colorectal cancer (mCRC) treated with regorafenib (REG) who had progression-free survival (PFS) $>4$ months (m): Subgroup analysis of the phase 3 CORRECT trial. Ann Oncol 27 (Suppl 6): S516P, 2016.

26. Puthiamadathil JM and Weinberg BA: Emerging combination therapies for metastatic colorectal cancer - impact of trifluridine/tipiracil. Cancer Manag Res 9: 461-469, 2017.

27. Austin PC: The performance of different propensity score methods for estimating marginal hazard ratios. Stat Med 32: 2837-2849, 2013.

This work is licensed under a Creative Commons Attribution-NonCommercial-NoDerivatives 4.0 International (CC BY-NC-ND 4.0) License. 